\title{
Effect of Habitat Manipulation on Infestation of Paddy Leaf Folder, Cnaphalocrocis medinalis (Guenee)
}

\author{
S.D. Desai", R. Swaminathan and V.S. Desai \\ Department of Agricultural Entomology, College of Agriculture, Dapoli, Dr. Balasaheb Sawant \\ Konkan Krishi Vidyapeeth, Dapoli, Dist. Ratnagiri-415712 (M.S.), India \\ *Corresponding author
}

A B S T R A C T

\begin{tabular}{|c|c|}
\hline & \\
\hline $\begin{array}{l}\text { Leaf fo } \\
\text { Cnaph ha } \\
\text { medina } \\
\text { Mustar } \\
\text { Ekdan } \\
\text { Marigo } \\
\text { Zinnia. }\end{array}$ & \multirow{3}{*}{$\begin{array}{l}\text { The present investigation was undertaken during Kharif } 2015 \text { and Kharif } \\
2016 \text { to study the effect of habitat manipulation on infestation of rice leaf } \\
\text { folder, Cnaphalocrocis medinalis (Guenee) under field condition. The leaf } \\
\text { folder infestation was maximum in SMW 32, thereafter it gradually } \\
\text { decreased up to SMW } 36 \text { and was in the range of } 0.15 \text { to } 1.49 \text { and } 0.05 \text { to } \\
1.80 \text { during both the years. The treatments Sunflower, Mustard, Sesame, } \\
\text { Ekdandi, Cock's comb, Marigold, Cosmos and Zinnia as flowering plants } \\
\text { on rice bunds were found best for reducing the rice leaf folder infestation in } \\
\text { both the years. }\end{array}$} \\
\hline Article Info & \\
\hline & \\
\hline
\end{tabular}

\section{Introduction}

Rice (Oryza sativa L.) is the most important staple food grain crop of the world which constitutes the principle food for about 60 per cent of the world's population and about 65 per cent population in India. It is the most important among the rainfed crops grown in the country. In the world, rice is cultivated on about 163.1 million hectares with a production of 722.5 million tons and productivity of 4.4 tons $\mathrm{ha}^{-1}$ (Anonymous, 2012). The India's rice production reached to a record high of 104.32 million tons from an area of 43.17 million hectares with productivity of 2.42 tons $\mathrm{ha}^{-1}$ in 2011-2012 (Anonymous, 2013). Though the production is high, the hectare $^{-1}$ yield is very poor as compared to other rice growing countries like Spain, Japan, Australia and China. The main reasons for low productivity are vagaries of nature, low fertilizer use efficiency, poor management, and heavy infestation of weeds, diseases and pests.

The warm and humid climatic condition being conducive for many pests, form a major constraint for increasing rice production in this ecosystem. Only 18-20 species out of nearly 800 insect species recorded on rice are major pests in tropical Asia. Few pests like gall midge, stem borer, leaf folder and brown plant hopper are of major significance limiting rice production. The average yield 
losses in rice have been estimated to vary between 21-51 per cent (Krishnaiah and Verma, 2012).

In view of reducing the unnecessary insecticide use, promotion of natural biological control ecosystem services through enriching bunds and other surrounding areas with flowering plants can significantly prevent outbreaks of rice pests. Such practices focus on biodiversity conservation, enhancing ecosystem services and avoiding pollution and will build resilience in production systems. In view of the conservation of natural enemies and thereby management of rice leaf folder in paddy ecosystem, the present research work was undertaken

\section{Materials and Methods}

A statistically designed field experiment using three replications, fifteen treatments (fourteen with flowering plants on bunds and one without flowering plants as control) was conducted during two kharif seasons of 2015 and 2016 at Agronomy Farm, College of Agriculture, Dapoli to evaluate the effect of flowering plants for conservation of the natural enemies in rice ecosystem and their impact on incidence of rice pests.

Rice Variety: Ratnagiri-1

Design: R.B.D.

Replications: Three

Bund size for each treatment: $10 \mathrm{~m}$ length and 1m wide

Distance between two plots: $2 \mathrm{~m}$

Distance between two flowering plants: 30 $\mathrm{cm} \times 30 \mathrm{~cm}$

Plot size (each treatment): $10 \mathrm{mx} 10 \mathrm{~m}$

\section{Planting of flowering plants on the bunds}

Thirty to thirty-five days old nursery raised seedlings of all the flowering plants were ready to transfer in rice ecosystem. The nursery raised flowering plants in the polythene bags were planted on the rice bunds at a distance of $30 \mathrm{~cm} \times 30 \mathrm{~cm}$. Three rows of flowering plants were planted by keeping $0.30 \mathrm{~m}$ distance between two rows on rice bunds for $10 \mathrm{~m}$ length

\section{Method of recording observations}

The total leaves per hill were counted and the numbers of leaves damaged by paddy leaf folder were counted from the same hill. The per cent infestation by leaf folder per hill was calculated by using following equation:

Infestation of

Number of damaged leaves

Leaf folder $(\%)$ = ----------------------- X 100

Total number of leaves

Data on per cent leaf folder infestation were converted into arc sine transformation and subjected to statistical analysis. The original data was then retransformed and used for interpretation.

\section{Record of natural enemies in paddy ecosystem}

The same ten rice hills randomly selected for recording infestation of insect pests of paddy from each plot having different flowering plants on bunds were used for recording the presence of natural enemies on rice hills. Different natural enemies were recorded at weekly interval according to Standard Meteorological Week (SMW). Similarly, ten flowering plants on bunds were randomly selected for recording the natural enemies. The natural enemies were recorded up to the harvest of the crop. The natural enemy viz., 
damselfly, dragonfly, syrphid fly, tachinid fly, bombylid fly, predatory wasps and spiders were counted from each rice hill or flowering plant. Data were converted to square root transformation $(\sqrt{ } \mathrm{n}+0.5)$ and subjected to statistical analysis.

\section{Results and Discussion}

Impact of habitat manipulation on rice leaf folder, Cnaphalocrocis medinalis (Guenee) during Kharif 2015and Kharif 2016

The data on the per cent infestation of leaf folder during Kharif 2015 is presented in Table 1. The leaf folder infestation was maximum in SMW 32, thereafter it gradually decreased up to SMW 36 (Table 1). It was in the range of 0.15 to 1.49 . The treatments Sunflower, Mustard, Sesamum, Ekdandi, Cock's comb, Marigold, Cosmos, Zinnia and Niger as flowering plants on rice bunds were found best for reducing the rice leaf folder infestation and recorded $0.15,0.16,0.19$, $0.24,0.30,0.34,0.38,0.41$ and 0.61 per cent infestation respectively and all these treatments were significantly superior over rest of the treatments and at par with each other. The maximum seasonal mean per cent infestation (1.49) was recorded in control where no flowering plants were on bund.

The data on per cent infestation of leaf folder during Kharif 2016 is presented in Table 2. The seasonal mean per cent infestation of rice leaf folder was in the range of 0.05 to 1.80 . The treatments Sunflower, Mustard, Ekdandi, Marigold, Sesamum, Zinnia, Cosmos and Cock's comb as flowering plants on rice bunds were found to be the best treatments for reducing the rice leaf folder infestation by recording $0.05,0.14,0.14,0.18,0.22,0.28$, 0.41 and 0.43 per cent infestation, respectively and all these treatments were significantly superior over rest of the treatments but at par with each other.
The results of the present findings are corroborative with results of other workers. Alvi et al., (2003) reported the peak in the third week of September, 2000 (4.63 larvae/ hill) and September, 2001 (4.41 larvae/ hill), which later decreased and finally disappeared in the second week of October. Subir et al., (2006) showed that the pest appeared in August and was recorded throughout the season. Ahmad et al., (2010) reported that infested leaves were observed in the field from $27^{\text {th }}$ standard week that reached to maximum (5.3\%/10 hills) during $37^{\text {th }}$ standard week. Chakraborty and Deb (2011) reported that the leaf folder population initiated during $25^{\text {th }}$ standard meteorological week that increased slowly up to $27^{\text {th }}$ SMW and was steady up to $32^{\text {nd }}$ SMW with the maximum during $34^{\text {th }}$ SMW. Wagh (2014) revealed that the per cent infestation of leaf folder in different treatments of nectar rich plants (Aster, Sesame, Petunia, Sunflower, Cufia, Marigold, Niger as border plants and without border) ranged from 3.572 to 8.493 . The infestation gradually decreased from the $2^{\text {nd }}$ week of September to week $1^{\text {st }}$ week of October and disappearing thereafter. The variation in results may be due to difference in climatic conditions of different areas.

Evaluation of flowering plants for the activity of natural enemies during Kharif 2015 and Kharif 2016

Among the natural enemies, damselflies and dragonflies (Odonata) were recorded during the $32^{\text {nd }}$ to $40^{\text {th }}$ SMW on paddy as well as the flowering plants on the bunds during kharif 2015 and 2016 (Tables 3 and 4) The maximum population of damselflies was recorded on paddy farmscaped with marigold (1.42) and cock's comb (1.78) during 2015 and 2016 kharif seasons, respectively; whereas, their population on the flowering plants was the maximum on cock's comb $(0.81)$, followed by that on marigold $(0.62)$. 
Table.1 Comparative infestation of paddy leaf folder under farmscape treatments at Dapoli (MS) during kharif, 2015

\begin{tabular}{|c|c|c|c|c|c|c|}
\hline \multirow[b]{2}{*}{ Treatments } & \multicolumn{6}{|c|}{ Mean infestation (\%) in different SMW } \\
\hline & SMW 32 & SMW 33 & SMW 34 & SMW 35 & SMW 36 & Seasonal mean $(\%)$ \\
\hline $\mathrm{T}_{1}$ Marigold & $3.26(0.32)^{*}$ & $3.10(0.29)$ & $4.23(0.54)$ & $3.45(0.36)$ & $1.93(0.11)$ & $3.36(0.34)$ \\
\hline $\mathrm{T}_{2}$ Niger & $5.98(1.08)$ & $4.30(0.56)$ & $4.75(0.69)$ & $3.79(0.44)$ & $2.91(0.26)$ & $4.47(0.61)$ \\
\hline $\mathrm{T}_{3}$ Mustard & $2.13(0.14)$ & $1.78(0.10)$ & $3.25(0.32)$ & $1.46(0.06)$ & $1.76(0.09)$ & $2.28(0.16)$ \\
\hline $\mathrm{T}_{4}$ Sesamum & $3.01(0.27)$ & $2.49(0.19)$ & $2.59(0.20)$ & $2.43(0.18)$ & $1.80(0.10)$ & $2.49(0.19)$ \\
\hline $\mathrm{T}_{5}$ Sunflower & $2.77(0.23)$ & $2.19(0.15)$ & $2.33(0.16)$ & $1.98(0.12)$ & $1.72(0.09)$ & $2.23(0.15)$ \\
\hline $\mathrm{T}_{6} \operatorname{Cosmos}$ & $4.88(0.72)$ & $2.95(0.27)$ & $3.48(0.37)$ & $3.04(0.28)$ & $2.25(0.15)$ & $3.55(0.38)$ \\
\hline $\mathrm{T}_{7}$ Gomphrina & $8.06(1.97)$ & $6.03(1.10)$ & $6.05(1.11)$ & $5.14(0.80)$ & $3.66(0.41)$ & $5.97(1.08)$ \\
\hline $\mathrm{T}_{8}$ Ekdandi & $4.90(0.73)$ & $1.89(0.11)$ & $0.18(0.0)$ & $2.09(0.13)$ & $2.22(0.15)$ & $2.79(0.24)$ \\
\hline $\mathrm{T}_{9}$ Cock's comb & $3.80(0.44)$ & $2.83(0.24)$ & $2.60(0.21)$ & $3.58(0.39)$ & $2.35(0.17)$ & $3.13(0.30)$ \\
\hline $\mathrm{T}_{10}$ Calendula & $7.51(1.71)$ & $5.60(0.95)$ & $5.83(1.03)$ & $4.53(0.62)$ & $4.21(0.54)$ & $5.66(0.97)$ \\
\hline$T_{11}$ Harana & $7.52(1.71)$ & $6.13(1.14)$ & $5.94(1.07)$ & $4.36(0.58)$ & $4.57(0.64)$ & $5.82(1.03)$ \\
\hline $\mathrm{T}_{12 .}$.Balsam & $8.24(2.05)$ & $6.36(1.23)$ & $6.30(1.20)$ & $4.97(0.75)$ & $4.29(0.56)$ & $6.19(1.16)$ \\
\hline $\mathrm{T}_{13}$ Blackgram & $8.25(2.06)$ & $6.44(1.26)$ & $6.32(1.21)$ & $5.30(0.85)$ & $4.31(0.56)$ & $6.26(1.19)$ \\
\hline $\mathrm{T}_{14}$ Zinnia & $5.63(0.96)$ & $3.73(0.42)$ & $3.22(0.32)$ & $2.06(0.13)$ & $1.86(0.11)$ & $3.66(0.41)$ \\
\hline $\mathrm{T}_{15}$ Control & $9.33(2.63)$ & $7.23(1.58)$ & $7.04(1.5)$ & $5.76(1.01)$ & $4.92(0.74)$ & $7.02(1.49)$ \\
\hline S. E \pm & 0.92 & 0.99 & 0.90 & 0.98 & 0.82 & 0.85 \\
\hline $\mathrm{CD}$ at $5 \%$ & 2.66 & 2.87 & 2.60 & NS & 2.38 & 2.47 \\
\hline
\end{tabular}

\footnotetext{
* Figures in parentheses are re-transformed percent values SMW- Standard Meteorological Week
} 
Table.2 Comparative infestation by paddy leaf folder under farmscape treatments at Dapoli (MS) during kharif, 2016

\begin{tabular}{|c|c|c|c|c|c|c|}
\hline \multirow{2}{*}{ Treatments } & \multicolumn{6}{|c|}{ Mean infestation (\%) in different SMW } \\
\hline & SMW 32 & SMW 33 & SMW 34 & SMW 35 & SMW 36 & Seasonal mean $(\%)$ \\
\hline $\mathrm{T}_{1}$ Marigold & $3.01(0.28)^{*}$ & $0.18(0.0)$ & $3.18(0.31)$ & $2.44(0.18)$ & $2.23(0.15)$ & $2.46(0.18)$ \\
\hline $\mathrm{T}_{2}$ Niger & $6.07(1.12)$ & $6.11(1.13)$ & $5.04(0.77)$ & $3.14(0.30)$ & $3.62(0.40)$ & $4.97(0.75)$ \\
\hline $\mathrm{T}_{3}$ Mustard & $3.75(0.43)$ & $0.18(0.0)$ & $1.600(0.08)$ & $1.54(0.07)$ & $1.37(0.06)$ & $2.16(0.14)$ \\
\hline $\mathrm{T}_{4}$ Sesamum & $4.33(0.57)$ & $0.18(0.0)$ & $1.97(0.12)$ & $2.49(0.19)$ & $2.50(0.19)$ & $2.66(0.22)$ \\
\hline $\mathrm{T}_{5}$ Sunflower & $0.18(0.0)$ & $2.18(0.14)$ & $0.18(0.0)$ & $2.00(0.12)$ & $0.18(0.0)$ & $1.34(0.05)$ \\
\hline $\mathrm{T}_{6} \operatorname{Cosmos}$ & $3.59(0.39)$ & $2.75(0.23)$ & $4.32(0.57)$ & $4.16(0.53)$ & $2.55(0.20)$ & $3.65(0.41)$ \\
\hline $\mathrm{T}_{7}$ Gomphrina & $8.39(2.13)$ & $6.70(1.36)$ & $6.74(1.38)$ & $5.44(0.90)$ & $3.99(0.48)$ & $6.42(1.25)$ \\
\hline $\mathrm{T}_{8}$ Ekdandi & $2.76(0.23)$ & $2.35(0.17)$ & $0.18(0.0)$ & $2.47(0.19)$ & $2.09(0.13)$ & $2.18(0.14)$ \\
\hline $\mathrm{T}_{9}$ Cock's comb & $5.27(0.85)$ & $4.44(0.60)$ & $3.01(0.27)$ & $2.53(0.20)$ & $1.98(0.12)$ & $3.74(0.43)$ \\
\hline $\mathrm{T}_{10}$ Calendula & $7.94(1.91)$ & $6.09(1.13)$ & $5.76(1.01)$ & $5.35(0.87)$ & $4.52(0.62)$ & $6.04(1.11)$ \\
\hline$T_{11}$ Harana & $9.46(2.70)$ & $6.13(1.14)$ & $6.84(1.42)$ & $5.41(0.89)$ & $5.04(0.77)$ & $6.76(1.38)$ \\
\hline $\mathrm{T}_{12}$. Balsam & $9.74(2.86)$ & $6.35(1.22)$ & $6.05(1.11)$ & $5.65(0.97)$ & $4.43(0.60)$ & $6.68(1.36)$ \\
\hline $\mathrm{T}_{13}$ Blackgram & $8.86(2.37)$ & $7.51(1.71)$ & 7.35 (1.64) & $5.54(0.93)$ & $5.17(0.81)$ & $7.02(1.49)$ \\
\hline $\mathrm{T}_{14}$ Zinnia & $2.68(0.22)$ & $3.69(0.41)$ & $3.96(0.48)$ & $1.72(0.09)$ & $1.34(0.06)$ & $3.01(0.28)$ \\
\hline $\mathrm{T}_{15}$ Control & 9.97 (3.00) & $8.15(2.01)$ & 7.78 (1.83) & 6.39 (1.24) & $5.47(0.91)$ & $7.71(1.80)$ \\
\hline S. E \pm & 1.13 & 0.69 & 0.89 & 0.99 & 0.87 & 0.85 \\
\hline $\mathrm{CD}$ at $5 \%$ & 3.27 & 2.01 & 2.59 & 2.86 & 2.51 & 2.47 \\
\hline
\end{tabular}

* Figures in parentheses are re-transformed per cent values. SMW- Standard Meteorological Week 
Table.3 Effect of farmscape plants on the activity of natural enemies of pests of Rice (kharif -2015)

\begin{tabular}{|c|c|c|c|c|c|c|c|}
\hline $\begin{array}{l}\text { Farmscape } \\
\text { Treatments }\end{array}$ & $\begin{array}{c}\text { Damsel } \\
\text { flies }\end{array}$ & $\begin{array}{c}\text { Dragon } \\
\text { flies }\end{array}$ & $\begin{array}{c}\text { Predatory } \\
\text { wasps }\end{array}$ & $\begin{array}{l}\text { Syrphid } \\
\text { flies }\end{array}$ & $\begin{array}{l}\text { Tachinid } \\
\text { flies }\end{array}$ & $\begin{array}{l}\text { Bombylid } \\
\text { flies }\end{array}$ & Spiders \\
\hline $\mathrm{T}_{1}$ Marigold & $\begin{array}{c}3.94 \\
(15.05)\end{array}$ & $\begin{array}{c}3.64 \\
(12.77)\end{array}$ & $\begin{array}{c}4.09 \\
(16.19)\end{array}$ & $\begin{array}{l}2.15 \\
(4.1)\end{array}$ & $\begin{array}{c}2.48 \\
(5.63)\end{array}$ & $\begin{array}{c}2.83 \\
(7.49)\end{array}$ & $\begin{array}{c}4.89 \\
(23.38)\end{array}$ \\
\hline $\mathrm{T}_{2}$ Niger & $\begin{array}{c}3.70 \\
(13.21)\end{array}$ & $\begin{array}{c}3.30 \\
(10.37)\end{array}$ & $\begin{array}{c}3.97 \\
(15.25)\end{array}$ & $\begin{array}{l}2.64 \\
(6.49)\end{array}$ & $\begin{array}{l}3.17 \\
(9.53)\end{array}$ & $\begin{array}{c}3.01 \\
(8.54)\end{array}$ & $\begin{array}{c}4.63 \\
(20.93)\end{array}$ \\
\hline $\mathrm{T}_{3}$ Mustard & $\begin{array}{c}3.95 \\
(15.09)\end{array}$ & $\begin{array}{c}3.27 \\
(10.20)\end{array}$ & $\begin{array}{c}2.96 \\
(8.24)\end{array}$ & $\begin{array}{c}2.36 \\
(5.08)\end{array}$ & $\begin{array}{c}2.78 \\
(7.20)\end{array}$ & $\begin{array}{c}2.63 \\
(6.39)\end{array}$ & $\begin{array}{c}4.48 \\
(19.57)\end{array}$ \\
\hline $\mathrm{T}_{4}$ Sesame & $\begin{array}{c}2.89 \\
(7.85)\end{array}$ & $\begin{array}{l}3.10 \\
(9.13)\end{array}$ & $\begin{array}{c}3.12 \\
(9.24)\end{array}$ & $\begin{array}{c}2.58 \\
(6.17)\end{array}$ & $\begin{array}{l}2.16 \\
(4.15)\end{array}$ & $\begin{array}{c}2.25 \\
(4.58)\end{array}$ & $\begin{array}{c}4.59 \\
(20.59)\end{array}$ \\
\hline $\mathrm{T}_{5}$ Sunflower & $\begin{array}{c}4.01 \\
(15.61) \\
\end{array}$ & $\begin{array}{l}2.74 \\
(7.01)\end{array}$ & $\begin{array}{c}4.35 \\
(18.44) \\
\end{array}$ & $\begin{array}{c}2.13 \\
(4.03) \\
\end{array}$ & $\begin{array}{c}2.55 \\
(5.98) \\
\end{array}$ & $\begin{array}{c}3.06 \\
(8.88) \\
\end{array}$ & $\begin{array}{c}3.74 \\
(13.47) \\
\end{array}$ \\
\hline $\mathrm{T}_{6}$ Cosmos & $\begin{array}{c}3.86 \\
(14.38)\end{array}$ & $\begin{array}{c}3.79 \\
(13.84)\end{array}$ & $\begin{array}{c}3.59 \\
(12.38)\end{array}$ & $\begin{array}{l}2.99 \\
(8.47)\end{array}$ & $\begin{array}{c}2.94 \\
(8.15)\end{array}$ & $\begin{array}{c}3.29 \\
(10.32)\end{array}$ & $\begin{array}{c}4.52 \\
(19.92)\end{array}$ \\
\hline $\mathrm{T}_{7}$ Gomphrena & $\begin{array}{c}3.79 \\
(13.88) \\
\end{array}$ & $\begin{array}{c}3.77 \\
(13.71) \\
\end{array}$ & $\begin{array}{c}3.73 \\
(13.42) \\
\end{array}$ & $\begin{array}{r}2.36 \\
(5.09) \\
\end{array}$ & $\begin{array}{l}2.01 \\
(3.54) \\
\end{array}$ & $\begin{array}{l}2.32 \\
(4.87) \\
\end{array}$ & $\begin{array}{c}5.08 \\
(25.26) \\
\end{array}$ \\
\hline$T_{8}$ Ekdandi & $\begin{array}{c}3.48 \\
(11.63)\end{array}$ & $\begin{array}{c}3.81 \\
(14.02)\end{array}$ & $\begin{array}{l}3.08 \\
(8.98) \\
\end{array}$ & $\begin{array}{c}1.84 \\
(2.89) \\
\end{array}$ & $\begin{array}{c}3.00 \\
(8.48)\end{array}$ & $\begin{array}{c}2.96 \\
(8.24)\end{array}$ & $\begin{array}{c}3.39 \\
(10.99)\end{array}$ \\
\hline $\mathrm{T}_{9}$ Cock'scomb & $\begin{array}{c}4.34 \\
(18.33)\end{array}$ & $\begin{array}{c}3.88 \\
(14.54)\end{array}$ & $\begin{array}{c}3.63 \\
(12.69)\end{array}$ & $\begin{array}{c}2.43 \\
(5.42) \\
\end{array}$ & $\begin{array}{l}2.63 \\
(6.41) \\
\end{array}$ & $\begin{array}{l}2.24 \\
(4.50)\end{array}$ & $\begin{array}{c}4.63 \\
(20.97)\end{array}$ \\
\hline $\mathrm{T}_{10}$ Calendula & $\begin{array}{c}3.71 \\
(13.25) \\
\end{array}$ & $\begin{array}{l}3.76 \\
(13.6) \\
\end{array}$ & $\begin{array}{c}3.69 \\
(13.13) \\
\end{array}$ & $\begin{array}{c}2.18 \\
(4.25) \\
\end{array}$ & $\begin{array}{c}2.69 \\
(6.72) \\
\end{array}$ & $\begin{array}{c}2.62 \\
(6.36) \\
\end{array}$ & $\begin{array}{c}5.14 \\
(25.94) \\
\end{array}$ \\
\hline$T_{11}$ Harna & $\begin{array}{c}3.58 \\
(12.32)\end{array}$ & $\begin{array}{c}2.83 \\
(7.49) \\
\end{array}$ & $\begin{array}{c}3.23 \\
(9.96) \\
\end{array}$ & $\begin{array}{l}2.40 \\
(5.27)\end{array}$ & $\begin{array}{l}2.85 \\
(7.62)\end{array}$ & $\begin{array}{c}2.36 \\
(5.07)\end{array}$ & $\begin{array}{c}4.44 \\
(19.2)\end{array}$ \\
\hline $\mathrm{T}_{12 .}$ Balsam & $\begin{array}{c}2.80 \\
(7.35) \\
\end{array}$ & $\begin{array}{c}1.99 \\
(3.45) \\
\end{array}$ & $\begin{array}{l}3.07 \\
(8.95) \\
\end{array}$ & $\begin{array}{c}2.71 \\
(6.86) \\
\end{array}$ & $\begin{array}{c}3.18 \\
(9.59) \\
\end{array}$ & $\begin{array}{c}2.13 \\
(4.04) \\
\end{array}$ & $\begin{array}{c}3.03 \\
(8.69) \\
\end{array}$ \\
\hline $\mathrm{T}_{13}$ Blackgram & $\begin{array}{c}3.20 \\
(9.71) \\
\end{array}$ & $\begin{array}{c}2.97 \\
(8.32) \\
\end{array}$ & $\begin{array}{c}3.40 \\
(11.08)\end{array}$ & $\begin{array}{l}2.54 \\
(5.97) \\
\end{array}$ & $\begin{array}{l}2.90 \\
(7.91) \\
\end{array}$ & $\begin{array}{c}2.23 \\
(4.48) \\
\end{array}$ & $\begin{array}{c}5.01 \\
(24.61) \\
\end{array}$ \\
\hline $\mathrm{T}_{14}$ Zinnia & $\begin{array}{c}3.71 \\
(13.25) \\
\end{array}$ & $\begin{array}{c}3.56 \\
(12.19) \\
\end{array}$ & $\begin{array}{c}3.55 \\
(12.13) \\
\end{array}$ & $\begin{array}{c}2.44 \\
(5.46) \\
\end{array}$ & $\begin{array}{l}2.05 \\
(3.71) \\
\end{array}$ & $\begin{array}{c}1.95 \\
(3.30) \\
\end{array}$ & $\begin{array}{c}5.17 \\
(26.26) \\
\end{array}$ \\
\hline $\mathrm{T}_{15}$ Control & $\begin{array}{c}2.0 \\
(3.50)\end{array}$ & $\begin{array}{l}2.38 \\
(5.16) \\
\end{array}$ & $\begin{array}{c}2.39 \\
(5.20 \mathrm{~S})\end{array}$ & $\begin{array}{c}1.68 \\
(2.32) \\
\end{array}$ & $\begin{array}{c}1.27 \\
(1.11) \\
\end{array}$ & $\begin{array}{c}1.56 \\
(1.95) \\
\end{array}$ & $\begin{array}{l}2.25 \\
(4.58)\end{array}$ \\
\hline S. E \pm & 0.32 & 0.33 & 0.42 & 0.37 & 0.39 & 0.45 & 0.48 \\
\hline $\mathrm{CD}$ at $5 \%$ & 0.93 & 0.96 & NS & NS & NS & NS & 1.38 \\
\hline
\end{tabular}

Figures in parentheses are re-transformed square values 
Table.4 Effect of farmscape plants on the activity of natural enemies of pests of Rice (kharif -2016)

\begin{tabular}{|c|c|c|c|c|c|c|c|}
\hline $\begin{array}{l}\text { Farmscape } \\
\text { Treatments }\end{array}$ & $\begin{array}{c}\text { Damsel } \\
\text { flies }\end{array}$ & $\begin{array}{c}\text { Dragon } \\
\text { flies }\end{array}$ & $\begin{array}{c}\text { Predatory } \\
\text { wasps }\end{array}$ & $\begin{array}{c}\text { Syrphid } \\
\text { flies }\end{array}$ & $\begin{array}{c}\text { Tachinid } \\
\text { flies }\end{array}$ & $\begin{array}{c}\text { Bombylid } \\
\text { flies }\end{array}$ & Spiders \\
\hline $\mathrm{T}_{1}$ Marigold & $\begin{array}{c}3.17 \\
(9.57)\end{array}$ & $\begin{array}{c}3.73 \\
(13.43)\end{array}$ & $\begin{array}{c}3.67 \\
(12.99)\end{array}$ & $\begin{array}{l}2.37 \\
(5.14)\end{array}$ & $\begin{array}{l}2.16 \\
(4.16)\end{array}$ & $\begin{array}{c}3.27 \\
(10.22)\end{array}$ & $\begin{array}{c}5.57 \\
(31.17)\end{array}$ \\
\hline $\mathrm{T}_{2}$ Niger & $\begin{array}{c}3.87 \\
(14.47)\end{array}$ & $\begin{array}{c}4.26 \\
(17.67)\end{array}$ & $\begin{array}{c}4.17 \\
(16.91)\end{array}$ & $\begin{array}{l}2.70 \\
(6.79)\end{array}$ & $\begin{array}{c}2.58 \\
(6.18)\end{array}$ & $\begin{array}{c}2.37 \\
(5.10)\end{array}$ & $\begin{array}{c}4.00 \\
(15.53)\end{array}$ \\
\hline $\mathrm{T}_{3}$ Mustard & $\begin{array}{c}4.08 \\
(16.11)\end{array}$ & $\begin{array}{l}3.94 \\
(15.0)\end{array}$ & $\begin{array}{c}4.06 \\
(15.99)\end{array}$ & $\begin{array}{c}1.94 \\
(3.25)\end{array}$ & $\begin{array}{c}2.44 \\
(5.43)\end{array}$ & $\begin{array}{c}2.52 \\
(5.85)\end{array}$ & $\begin{array}{c}4.79 \\
(22.48)\end{array}$ \\
\hline $\mathrm{T}_{4}$ Sesame & $\begin{array}{c}3.51 \\
(11.83)\end{array}$ & $\begin{array}{c}2.96 \\
(8.25)\end{array}$ & $\begin{array}{l}2.97 \\
(8.32)\end{array}$ & $\begin{array}{c}1.87 \\
(3.02)\end{array}$ & $\begin{array}{c}3.52 \\
(11.91)\end{array}$ & $\begin{array}{c}2.38 \\
(5.17)\end{array}$ & $\begin{array}{c}5.33 \\
(27.89)\end{array}$ \\
\hline $\mathrm{T}_{5}$ Sunflower & $\begin{array}{l}3.18 \\
(9.59)\end{array}$ & $\begin{array}{c}2.93 \\
(8.06)\end{array}$ & $\begin{array}{c}3.90 \\
(14.74)\end{array}$ & $\begin{array}{c}2.78 \\
(7.22)\end{array}$ & $\begin{array}{c}3.20 \\
(9.76)\end{array}$ & $\begin{array}{c}2.62 \\
(6.35)\end{array}$ & $\begin{array}{c}4.66 \\
(21.22)\end{array}$ \\
\hline $\mathrm{T}_{6}$ Cosmos & $\begin{array}{c}3.28 \\
(10.25)\end{array}$ & $\begin{array}{l}2.96 \\
(8.26)\end{array}$ & $\begin{array}{c}4.06 \\
(15.99)\end{array}$ & $\begin{array}{l}2.40 \\
(5.27)\end{array}$ & $\begin{array}{c}2.51 \\
(5.79)\end{array}$ & $\begin{array}{c}3.93 \\
(14.93)\end{array}$ & $\begin{array}{c}5.11 \\
(25.57)\end{array}$ \\
\hline $\mathrm{T}_{7}$ Gomphrena & $\begin{array}{c}4.16 \\
(16.82) \\
\end{array}$ & $\begin{array}{c}3.72 \\
(13.3) \\
\end{array}$ & $\begin{array}{c}3.37 \\
10.83) \\
\end{array}$ & $\begin{array}{c}2.15 \\
(4.11) \\
\end{array}$ & $\begin{array}{c}2.47 \\
(5.61) \\
\end{array}$ & $\begin{array}{l}2.24 \\
(4.52) \\
\end{array}$ & $\begin{array}{c}5.63 \\
(30.13) \\
\end{array}$ \\
\hline$T_{8}$ Ekdandi & $\begin{array}{c}3.73 \\
(13.43)\end{array}$ & $\begin{array}{c}3.94 \\
(15.01)\end{array}$ & $\begin{array}{c}3.60 \\
(12.47)\end{array}$ & $\begin{array}{l}2.45 \\
(5.49)\end{array}$ & $\begin{array}{c}2.60 \\
(6.28)\end{array}$ & $\begin{array}{c}2.61 \\
(6.33)\end{array}$ & $\begin{array}{c}5.16 \\
(26.15)\end{array}$ \\
\hline $\mathrm{T}_{9}$ Cock'scomb & $\begin{array}{l}4.63( \\
20.96)\end{array}$ & $\begin{array}{c}3.55 \\
(12.07)\end{array}$ & $\begin{array}{c}2.86 \\
(7.68) \\
\end{array}$ & $\begin{array}{c}2.45 \\
(5.52) \\
\end{array}$ & $\begin{array}{l}2.74 \\
(7.0)\end{array}$ & $\begin{array}{c}2.91 \\
(7.99)\end{array}$ & $\begin{array}{c}5.44 \\
(29.04)\end{array}$ \\
\hline $\mathrm{T}_{10}$ Calendula & $\begin{array}{c}3.95 \\
(15.1) \\
\end{array}$ & $\begin{array}{c}2.73 \\
(6.97) \\
\end{array}$ & $\begin{array}{c}3.54 \\
(12.01)\end{array}$ & $\begin{array}{c}2.34 \\
(4.97) \\
\end{array}$ & $\begin{array}{c}2.94 \\
(8.11) \\
\end{array}$ & $\begin{array}{c}2.63 \\
(6.41) \\
\end{array}$ & $\begin{array}{c}4.99 \\
(24.35) \\
\end{array}$ \\
\hline$T_{11}$ Harna & $\begin{array}{c}3.87 \\
(14.47)\end{array}$ & $\begin{array}{c}2.66 \\
(6.56)\end{array}$ & $\begin{array}{c}3.05 \\
(8.83) \\
\end{array}$ & $\begin{array}{c}2.10 \\
(3.93) \\
\end{array}$ & $\begin{array}{c}2.50 \\
(5.77)\end{array}$ & $\begin{array}{c}2.57 \\
(6.13) \\
\end{array}$ & $\begin{array}{l}3.09 \\
(9.04)\end{array}$ \\
\hline $\mathrm{T}_{12} \cdot$ Balsam & $\begin{array}{c}3.02 \\
(8.63) \\
\end{array}$ & $\begin{array}{c}2.90 \\
(7.88)\end{array}$ & $\begin{array}{c}3.34 \\
(10.65)\end{array}$ & $\begin{array}{c}2.85 \\
(7.62)\end{array}$ & $\begin{array}{c}1.69 \\
(2.37) \\
\end{array}$ & $\begin{array}{c}2.10 \\
(3.91) \\
\end{array}$ & $\begin{array}{c}3.83 \\
(14.18)\end{array}$ \\
\hline $\mathrm{T}_{13}$ Blackgram & $\begin{array}{c}3.39 \\
(10.99)\end{array}$ & $\begin{array}{c}3.57 \\
(12.27)\end{array}$ & $\begin{array}{c}3.46 \\
(11.48)\end{array}$ & $\begin{array}{c}3.08 \\
(8.99) \\
\end{array}$ & $\begin{array}{l}3.08 \\
(8.97) \\
\end{array}$ & $\begin{array}{l}3.20 \\
(9.72)\end{array}$ & $\begin{array}{c}4.41 \\
(18.98)\end{array}$ \\
\hline $\mathrm{T}_{14}$ Zinnia & $\begin{array}{c}3.58 \\
(12.29) \\
\end{array}$ & $\begin{array}{c}3.32 \\
(10.5) \\
\end{array}$ & $\begin{array}{c}3.89 \\
(14.63) \\
\end{array}$ & $\begin{array}{c}2.47 \\
(5.58) \\
\end{array}$ & $\begin{array}{c}2.42 \\
(5.37) \\
\end{array}$ & $\begin{array}{c}2.87 \\
(7.73) \\
\end{array}$ & $\begin{array}{c}4.74 \\
(21.97) \\
\end{array}$ \\
\hline $\mathrm{T}_{15}$ Control & $\begin{array}{l}2.29 \\
(4.73)\end{array}$ & $\begin{array}{c}2.56 \\
(6.06) \\
\end{array}$ & $\begin{array}{c}2.03 \\
(3.62) \\
\end{array}$ & $\begin{array}{c}1.36 \\
(1.34) \\
\end{array}$ & $\begin{array}{c}1.57 \\
(1.96) \\
\end{array}$ & $\begin{array}{c}1.32 \\
(1.24) \\
\end{array}$ & $\begin{array}{l}2.49 \\
(5.72)\end{array}$ \\
\hline S. E \pm & 0.33 & 0.28 & 0.31 & 0.19 & 0.18 & 0.21 & 0.45 \\
\hline $\mathrm{CD}$ at $5 \%$ & 0.94 & 0.82 & 0.90 & 0.55 & 0.51 & 0.62 & 1.30 \\
\hline
\end{tabular}

Figures in parentheses are re-transformed square values 


\section{Treatments Details}

\begin{tabular}{|c|l|l|l|}
\hline Tr. No. & Name of the plant & \multicolumn{1}{|c|}{ Botanical name } & \multicolumn{1}{c|}{ Family } \\
\hline $\mathrm{T}_{1}$ & Marigold & Tagetes erecta & Asteraceae \\
\hline $\mathrm{T}_{2}$ & Niger & Guizotia abyssinica & Asteraceae \\
\hline $\mathrm{T}_{3}$ & Mustard & Brassica rapa & Brassiceae \\
\hline $\mathrm{T}_{4}$ & Sesame & Sesame indicum & Pedaliaceae \\
\hline $\mathrm{T}_{5}$ & Sunflower & Helianthus annuus & Asteraceae \\
\hline $\mathrm{T}_{6}$ & Cosmos & Cosmos bipinnatus & Asteraceae \\
\hline $\mathrm{T}_{7}$ & Gomphrina & Gomphrena globose & Amaranthaceae \\
\hline $\mathrm{T}_{8}$ & Ekdandi & Tridex procumbens & Compositae(Asteraceae) \\
\hline $\mathrm{T}_{9}$ & Cock'scomb & Celosia argentea & Amaranthaceae \\
\hline $\mathrm{T}_{10}$ & Calendula & Calendula arvensis & Asteraceae \\
\hline $\mathrm{T}_{11}$ & Harna /Sontal & Senecio bombayensis & Asteraceae \\
\hline $\mathrm{T}_{12}$ & Balsam/Impatiens & Impatiens scapiflora & Balsaminaceae \\
\hline $\mathrm{T}_{13}$ & Black gram & Vigna mungo & Fabaceae \\
\hline $\mathrm{T}_{14}$ & Zinnia & Zinnia elegans & Asteraceae \\
\hline $\mathrm{T}_{15}$ & Control & - & - \\
\hline
\end{tabular}

As for dragonflies, their maximum population on paddy was recorded in the farmscape treatment with zinnia (1.15) and marigold (1.14) as the flowering plant on bunds during 2015 kharif season; while during kharif 2016, the maximum (1.73) dragonfly population on paddy was recorded when farmscaped with niger as flowering plants on bunds. On the flowering plants the maximum dragonfly population was noted on cosmos (0.68) during kharif, 2015, and on sesame (0.69) in kharif, 2016. The maximum numbers of predatory wasps were observed in the farmscape treatment with marigold (0.87) in 2015 and in the farmscape treatment with niger (0.89) during 2016.

On flowering plants, the maximum numbers of predatory wasps were observed on sunflower (1.40) during 2015 and on mustard (1.24) during the subsequent year in 2016. Syrphid flies happened to be in good numbers in the different farmscape treatments during 2015 their population being non-significant among the treatments, ranging from 0.17 to 0.53; whereas during 2016, significantly maximum syrphid flies were observed in the farmscape treatment with blackgram (0.65). On flowering plants the maximum syrphid flies were observed on niger (0.46), sesame (0.45) and zinnia (0.44) in 2015; likewise, on sunflower (0.55), Calendula (0.51) and zinnia (0.49) in the crop season of 2016. The occurrence of tachinid and bombyliid flies on paddy in the farmscape treatments did not differ significantly among the treatments during kharif 2015, while during kharif 2016, tachind flies were the maximum in the farmscape treatment with sunflower (0.83) and bombyliid flies in the treatment with cosmos (0.95). On the flowering plants their occurrence was more or less uniform and at par; however, they were recorded more on mustard and ekdandi during 2015, but on cosmos during 2016. The spider species recorded were: Argiope aemula (Araneidae), Oxyopes sp. (Oxyopidae) and Perenethis sp. (Pisauridae) during both the years. Relatively more numbers of spiders were recorded in the farmscape treatments with Calendula, Gomphrena and cock's comb, while on the flowering plants their numbers were more on blackgram, zinnia, marigold Gomphrena, cosmos, cock's comb, mustard and sesame, 
indicating that all such flowering plants are good sources to attract and conserve the spider population.

The leaf folder infestation was maximum in SMW 32, thereafter it gradually decreased up to SMW 36 and was in the range of 0.15 to 1.49 and 0.05 to 1.80 during both the years. The treatments Sunflower, Mustard, Sesame, Ekdandi, Cock's comb, Marigold, Cosmos and Zinnia as flowering plants on rice bunds were found best for reducing the rice leaf folder infestation in both the years. Considering the overall results of the twoyear study on impact of habitat manipulation on rice leaf folder it could be concluded that the flowering plants viz., Sunflower, Mustard, Sesame, Ekdandi, Cock's comb, Marigold, Cosmos and Zinnia attracted maximum number of natural enemies, thereby reducing pest infestation; therefore, can be recommended for habitat manipulation in rice eco-system to conserve natural enemies for rice leaf folder management

\section{References}

Ahmad, H., Khan, R. B., Sharma, D., Jamwal, V. V. S. and Gupta, S. 2010. Seasonal incidence, infestation and trap catches of Cnaphalocrocis medinalis (Guenee) in rice. Ann.Pl. Protec. Sci., 18 (2): 380383.
Alvi, S. M., Ali, M. A., Chaudhary, S. and Iqbal, S. 2003. Population trends and chemical control of rice leaf folder Cnaphalocrocis medinalis (Guenee) on rice crop. Int. J. Agri. Biol., 5(4): 615617.

Anonymous, 2013. Annu. Progress Rep. All India Coordinated Research Project on Weed Control, Dr. B.S.K.K.V., Dapoli Centre Jan to Dec. 2013, pp76-87.

Anonymous, 2012. htttps://www.eands.dacnet.nic.in.

Chakraborty, K., and Deb, D. C. 2011. Incidence of adult leaf folder, Cnaphalocrocis medinalis Guenee (Lepidoptera: Pyralidae) on Paddy crop in the agro climatic conditions of the northern parts of west Bengal, India. World J. Agric. Sci. 7 (6): 738-742.

Krishnaiah, K., and Verma N. R. G. 2012. Changing insect pest scenario in the rice ecosystem-A national perspective, www.rkmp.co.in.

Subir, R. M., Ahmed, S., Sahi, M. H. and Qadir, A. 2006. Pest weather interaction of major insect pests in rice ecosystem. SAARC J. Agric., 4: 213-220.

Wagh, P. D., 2014. Conservation of natural enemies in rice ecosystem and their impact on rice pests. M.Sc. (Ag) thesis Unpublished, Dr. B. S. K. K. V., Dapoli (Maharashtra) pp 82-90.

\section{How to cite this article:}

Desai, S.D., R. Swaminathan and Desai, V.S. 2017. Effect of Habitat Manipulation on Infestation of Paddy Leaf Folder, Cnaphalocrocis medinalis (Guenee). Int.J.Curr.Microbiol.App.Sci. 6(10): 1469-1477. doi: https://doi.org/10.20546/ijcmas.2017.610.174 